\title{
Archéopages
}

Archéopages

Archéologie et société

Hors-série 2 | 2010

Archéologie sans frontières

\section{Une coopération franco-chinoise : les fouilles du site de Gongying}

Alain Thote et Karine Michel

\section{(2) OpenEdition}

1 Journals

Édition électronique

URL : https://journals.openedition.org/archeopages/787

DOI : 10.4000/archeopages.787

ISSN : 2269-9872

Éditeur

INRAP - Institut national de recherches archéologiques préventives

Édition imprimée

Date de publication : 1 octobre 2010

Pagination : 79-84

ISSN : $1622-8545$

\section{Référence électronique}

Alain Thote et Karine Michel, « Une coopération franco-chinoise : les fouilles du site de Gongying »,

Archéopages [En ligne], Hors-série 2 | 2010, mis en ligne le 01 octobre 2010, consulté le 23 février 2023. URL : http://journals.openedition.org/archeopages/787 ; DOI : https://doi.org/10.4000/ archeopages.787 
Cleuziou S., 1985, « L'archéologie française à l'étranger : redéfinition ou déclin? » Les Nouvelles de l'Archéologie, ${ }^{\circ}$ 20, p. 8-25.

DUDAY H., 1995, « Anthropologie "de terrain", archéologie de la mort », in La Mort, passé, présent, conditionnel, colloque du Groupe vendéen d'études préhistoriques, La Roche-sur-Yon, 1994, GVEP, p. 33-58.

Leroi-Gourhan A., Bailloud G., Brezillon M., 1962, « L'hypogée II des Mournouards (Mesnil-sur-Oger, Marne) », Gallia Préhistoire, t. 5-1, p. 23-123.

LYONNET B., 2007, « La culture de Maikop, la Transcaucasie, l'Anatolie orientale et le Proche-Orient : relations et chronologie », in Lyonnet B. (DIR.), Les Cultures du Caucase (VI-III millénaire avant notre ère). Leurs relations avec le Proche-Orient, Paris, CNRs éditions, p. 133-161.

\section{Une coopération franco-chinoise: les fouilles du site de Gongying}

\author{
Alain Thote \\ École pratique des hautes études (4 ${ }^{e}$ section), chef de la mission \\ archéologique "Royaume de Chu », UMR 8155 " Centre \\ de recherches sur les civilisations de l'Asie orientale» \\ Karine Michel \\ Inrap, Grand-Est/Nord, Metz, céramologue
}

$\mathrm{H}$ istoire de l'archéologie chinoise. On connaît encore assez mal l'archéologie chinoise en France, en dépit de quelques expositions et d'articles qui se focalisent sur les sites les plus remarquables. En fait, c'est bien souvent l'aspect spectaculaire des découvertes que retient le public, tel qu'il est médiatisé par les journalistes qui en rendent compte. Pour comprendre ce qui se passe aujourd'hui en Chine dans ce domaine, une petite révolution, il convient de rappeler brièvement quelques faits. Très tôt, on s'y est intéressé à l'Antiquité, non pas avec la seule intention de collectionner de beaux objets, mais pour comprendre une société qui appartenait à une époque vue comme un âge d'or, et s'en inspirer. On a d'abord collationné les textes ; on les a publiés sous forme manuscrite, puis imprimés ; on les a annotés, commentés du début de l'Empire (221 avant notre ère) jusqu'à nos jours. Parallèlement, mais dans une bien moindre mesure, on s'est intéressé, dès le $\mathrm{XI}^{\mathrm{e}}$ siècle, aux vestiges matériels pour les étudier d'une façon scientifique. C'est à cette époque que parurent les premiers catalogues imprimés d'antiquités réunissant les plus belles pièces archéologiques connues. Certains de ces catalogues sont parvenus jusqu'à nous : chaque objet y est présenté par un dessin au trait et est accompagné d'une légende donnant ses dimensions, une description sommaire, son lieu de découverte, le nom de son propriétaire. On y reproduit encore son inscription, quand il en a une, sous deux formes : un estampage et une transcription en caractères standards, puisque les graphies avaient changé entre-temps.

Les missions scientifiques menées par les Occidentaux et les Japonais sur le sol chinois à partir de la fin du XIX ${ }^{\mathrm{e}}$ siècle, le pillage de ses trésors par des antiquaires peu scrupuleux alimentant le marché national et international durant la première moitié $\mathrm{du} \mathrm{xx}^{\mathrm{e}}$ siècle, ont eu deux effets importants sur l'archéologie de ce pays. D'une part, les Chinois ont entrepris, à leur tour, des fouilles à partir de 1928. Pour ce faire, ils ont choisi le site d'Anyang, la dernière capitale des Shang (environ $\mathrm{XVI}^{\mathrm{e}}$ siècle-1050 avant notre ère) et utilisé les méthodes qu'ils avaient apprises des Occidentaux. D'autre part, ils ont tenu à les entreprendre indépendamment de ces derniers. De 1949 jusqu'au début des années 1980, la Chine a développé son archéologie en vase clos, sans tenir compte des avancées faites ailleurs dans ce domaine. Les instructions données aux archéologues ont encadré et encadrent toujours, d'une manière rigide, leur travail. Elles sont uniformément appliquées sur tout le territoire : fouilles en carrés de $5 \times 5 \mathrm{~m}$, ou de $10 \times 10 \mathrm{~m}$, avec une berme d'un mètre de large mordant sur deux côtés, au nord et à l'est, chantiers strictement orientés au nord, etc. Ce sont, sous une forme plus systématique, les méthodes élaborées par sir Mortimer Wheeler (1890-1977) et ses prédécesseurs. D'autre part, pendant toutes ces années - et même encore aujourd'hui les recherches sont restées très dépendantes de la lecture que l'on faisait des sources textuelles anciennes, sur lesquelles se sont penchées des générations de lettrés. On tend d'abord à chercher dans les vestiges la confirmation de ce que l'on connaît par les textes classiques, les annales et les chroniques, sans toujours prendre en compte que ces sources ne sont pas nécessairement dignes de foi. L'archéologie a longtemps servi à illustrer ce que l'on connaît de l'histoire chinoise. Aujourd'hui c'est beaucoup moins le cas.

\section{L'archéologie chinoise aujourd'hui.}

L'ouverture économique de la Chine, à partir de 1978-79, a eu pour effet de modifier les conditions dans lesquelles sont opérées les fouilles. Les grands chantiers de travaux publics conduisent à faire d'innombrables découvertes, déclarées ou passées sous silence. Les échanges avec l'étranger ont permis aux archéologues chinois de mesurer le retard scientifique pris lorsque le pays était fermé à tout apport extérieur. Les moyens humains et financiers ont été améliorés dans le but de faire connaître la civilisation chinoise dans ses aspects les plus prestigieux. En particulier, on s'emploie aujourd'hui, à montrer que cette civilisation a un âge vénérable de cinq mille ans, à l'égal des plus grandes civilisations connues de par le monde.

On ne peut ici dresser un état des lieux: il resterait fort incomplet tant les changements récents sont importants, tout en restant inégaux selon les régions et les moyens mis en œuvre. De plus, la Chine a énormément investi dans la formation de jeunes archéologues, qui souvent la parachèvent en étudiant plusieurs années à l'étranger et surtout aux États-Unis. Elle place aussi des moyens considérables dans la recherche. La diffusion des connaissances est à la fois rapide et riche. Les publications chinoises d'aujourd'hui n'ont rien à nous envier : revues scientifiques à l'échelon national, provincial ou local, rapports complets de fouilles sous la forme de livres illustrés d'excellente qualité. En Chine aussi, on est passé d'une archéologie entièrement financée par l'État, 


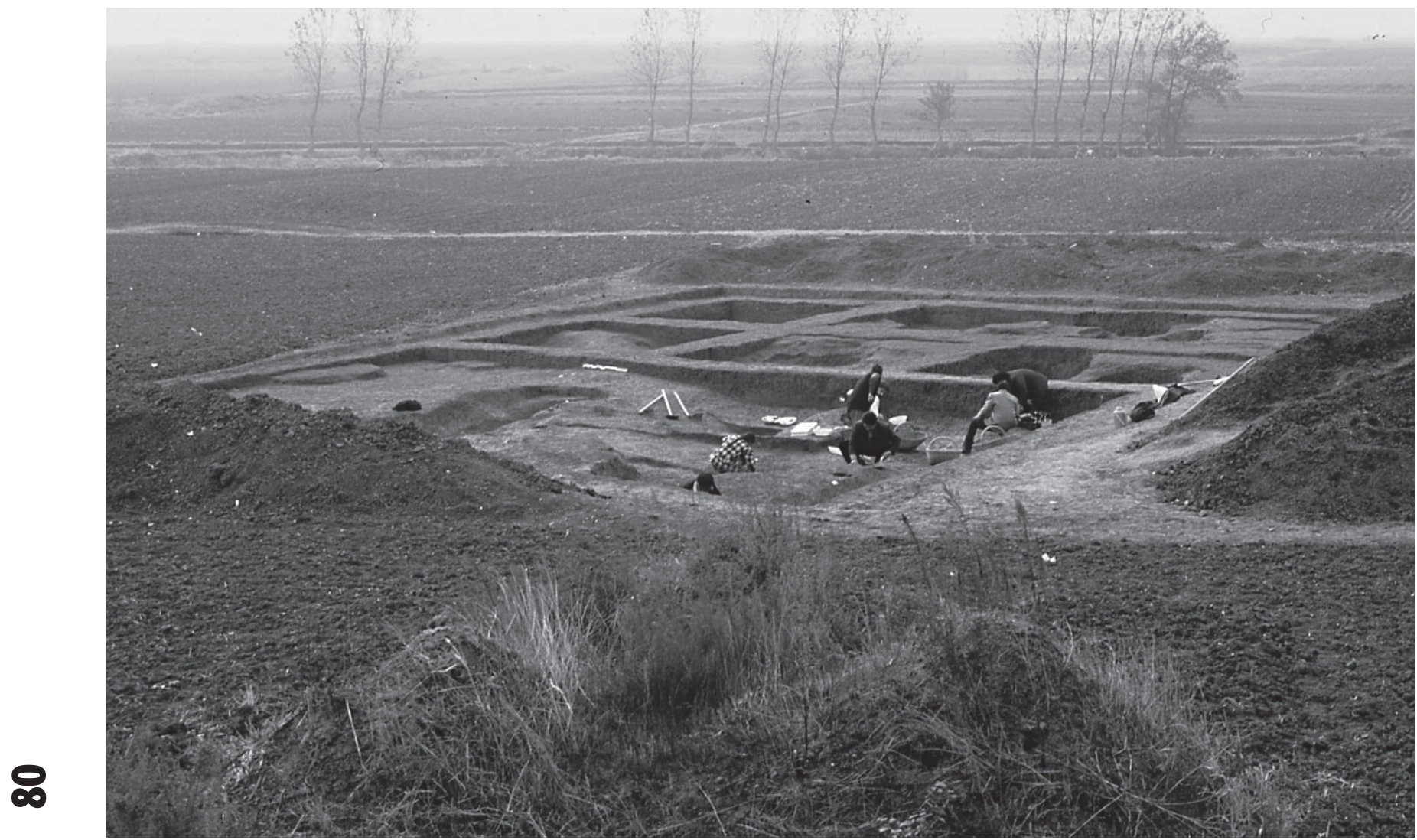

[Fig.1] Vue générale des fouilles menées lors des trois campagnes de 2000 2001 et 2002 . Au premier plan, la fouille française en aire ouverte, en arrière plan, la méthode Wheeler par carrés utilisée par les archéologues chinois.

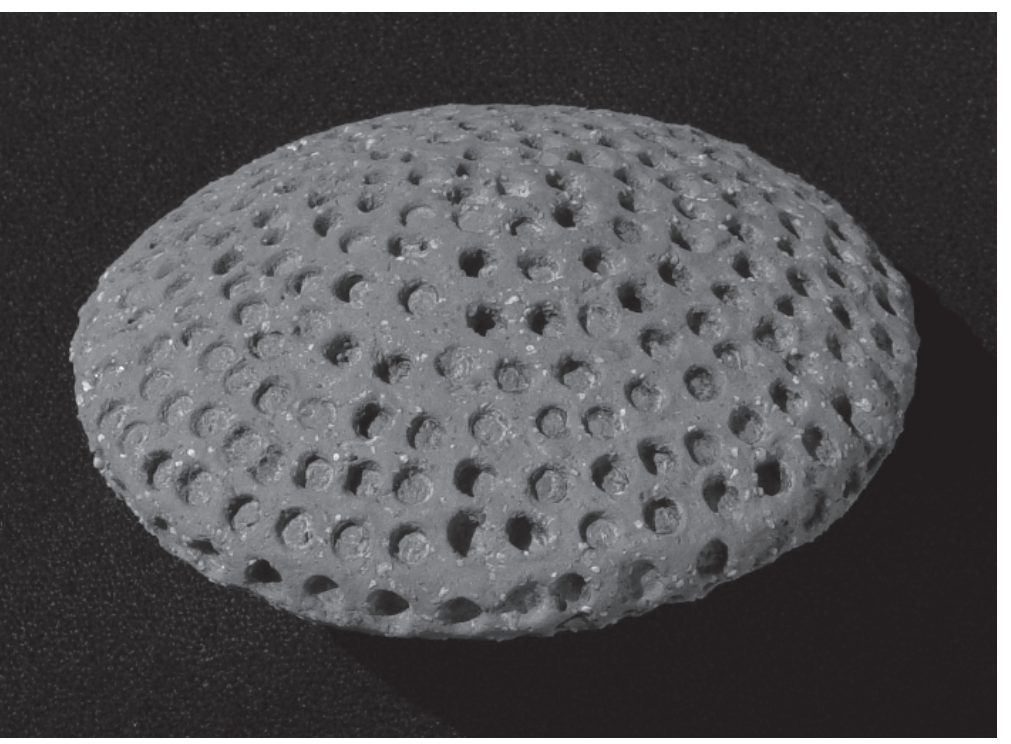

[Fig.2] Strigile chinois. Terre cuite, diamètre $7 \mathrm{~cm}$, épaisseur $3,2 \mathrm{~cm}$.

Forme ronde et profil lenticulaire.

Les deux faces, creusées d'une

multitude de petites alvéoles, rendent

la surface rugueuse. 
placée sous son entier contrôle, à une archéologie financée partiellement par les aménageurs. D’autre part, de nombreuses coopérations avec l'étranger ont été entreprises depuis la promulgation, en février 1991, d'une loi les autorisant. La décision d'entreprendre chacune de ces coopérations est mûrement réfléchie. Les premières fouilles menées dans ce cadre ont été faites dans la partie la plus occidentale de la Chine, le Xinjiang, par une équipe franco-chinoise, à partir de 1993. Puis, très rapidement, des coopérations ont été nouées, principalement avec les États-Unis et le Japon, mais aussi avec la plupart des pays européens, et avec l'Australie. Dans le cas général, il s'agit de prospections, d'études de matériel, de travaux de restauration. Les fouilles sont plus rares. En fait, dans ces coopérations, les Chinois tendent à privilégier les études de pointe dans des domaines techniques spécialisés qu'ils ne maîtrisent pas bien encore, notamment en anthropologie physique, en archéozoologie, etc. Aucune période n'est négligée, et la France, connue pour ses recherches sur le Paléolithique, a entrepris plusieurs coopérations avec la Chine.

L'origine de la mission « Royaume de Chu». Le programme de la mission archéologique « Royaume de Chu » a commencé en 1999 par une prospection dans le Henan, en partenariat avec l'institut archéologique de cette province et avec l'Université de Wuhan, sous la direction du professeur Yang Baocheng, qui a fouillé durant une vingtaine d'années à Anyang. Les institutions françaises impliquées dans le projet sont le Bureau du patrimoine - Centre d'archéologie du département de Seine-Saint-Denis, dirigé par M. Olivier Meyer, qui a mis d'importants moyens humains et matériels dans la mission ; le Centre de recherches sur les civilisations de l'Asie orientale (École pratique des hautes études/ CNRS/ Collège de France/Paris-Diderot-Paris 7), et cinq archéologues de l'Inrap, qui ont participé au travail de fouille et/ou de post-fouille ${ }^{\mathbf{1}}$ dont cinq semaines par convention. Le programme a reçu le soutien de l'École française d'Extrême-Orient et du service culturel et de coopération de l'ambassade de France en Chine, et il a été financé en majeure partie par le ministère des affaires étrangères.

\begin{tabular}{|c|c|}
\hline $\begin{array}{l}\text { archéologue, Bureau } \\
\text { du patrimoine } \\
\text { - Centre d'archéologie } \\
\text { du département de la } \\
\text { Seine-Saint-Denis (noté } \\
\text { par un astérisque après } \\
\text { le nom), Épinay-sur-Seine; } \\
\text { David Jouneau, } \\
\text { archéologue, responsable } \\
\text { d'opération au centre } \\
\text { archéologique Inrap de } \\
\text { Grand-Quevilly (Haute- } \\
\text { Normandie); Ivan Lafarge*, } \\
\text { archéologue, Épinay- } \\
\text { sur-Seine; Nicolas } \\
\text { Latsanopoulos*, } \\
\text { dessinateur, Épinay-sur- } \\
\text { Seine; Yann Le Jeune*, } \\
\text { archéologue, } \\
\text { géomorphologue et } \\
\text { géomaticien, ingénieur }\end{array}$ & $\begin{array}{l}\text { d'étude responsable de la } \\
\text { gestion de l'archéologie } \\
\text { en Vendée au service } \\
\text { régional de l'Archéologie } \\
\text { de la Dr AC Pays-de- } \\
\text { la-Loire; Benoît Leriche, } \\
\text { archéologue, } \\
\text { Inrap-Nord-Picardie; } \\
\text { Olivier Meyer*, directeur, } \\
\text { Épinay-sur-Seine; } \\
\text { Olivier Venture, maître } \\
\text { de conférences, École } \\
\text { pratique des hautes } \\
\text { études, } 4 \text { e section, } \\
\text { UMR } 8155 \text {, Paris; } \\
\text { Zhao Bing, chargé } \\
\text { de recherche, CNRS, } \\
\text { UMR } 8155 \text { « Centre } \\
\text { de recherches sur } \\
\text { les civilisations } \\
\text { de l'Asie Orientale». }\end{array}$ \\
\hline
\end{tabular}

Ce projet est né du constat que la fouille des tombes a été toujours privilégiée en Chine pour l'âge du Bronze (environ XVI ${ }^{\mathrm{e}}$ siècle221 avant notre ère), au point qu'aujourd'hui encore il est impossible de définir les différentes formes d'habitat de la période, encore moins d'en connaître l'évolution. Ni le plan des fermes ni l'aspect des greniers n'ont pu être restitués. Et tout ce qui a trait à la disposition des villages, aux relations entre l'homme et son environnement, à l'archéologie du paysage nous reste inconnu. Il apparaît que les méthodes de fouilles utilisées ont entravé les recherches dans ce domaine. La fouille en aire ouverte, l'enregistrement des vestiges découverts par unité stratigraphique, la prise en compte de l'intégralité de la céramique d'un site, associés à différentes méthodes d'analyse des données collectées sur les sites, permet d'aborder de plus amples questions que ne l'ont fait jusqu'à présent nos collègues chinois.

Le site de Gongying, sur lequel a porté notre travail, a été l'objet de trois campagnes de fouilles, assez brèves, l'une d'un mois en 2000, et les deux autres d'un mois et demi en 2001 et 2002. Puis quatre campagnes d'étude du matériel sur place, entre 2003 et 2005 , ont suivi. Le rapport complet sera publié en 2011 dans une édition bilingue franco-chinoise.

Présentation du site de Gongying. Le site de Gongying, au sud du village moderne du même nom, est bordé par une petite rivière, la Jian. En 200o, nous avons commencé par effectuer huit sondages disposés en croix, afin de choisir l'endroit le plus approprié pour les fouilles. Au cours des trois campagnes, un peu plus de $5 \%$ de la surface évaluée du site, soit environ $700 \mathrm{~m}^{2}$, ont été fouillés. Deux chantiers, l'un chinois, l'autre français, furent chaque fois mis en parallèle, une façon de tester les méthodes. Après une première campagne menée sur neuf carrés, on a adopté la fouille en aire ouverte sur le chantier français durant les deux années suivantes [Fig.1].

Le site de Gongying a d'abord été occupé dans la première moitié du deuxième millénaire (époque dite de Erlitou, un site majeur de la Chine centrale où apparaît la métallurgie du bronze), puis densément entre la fin du $\mathrm{IX}^{\mathrm{e}}$ siècle et le $\mathrm{v}^{\mathrm{e}}$ siècle avant notre ère, avant d'être abandonné pour être mis en culture au $\mathrm{III}^{\mathrm{e}}-\mathrm{II}^{\mathrm{e}}$ siècle avant notre ère. Les vestiges se composent, comme sur la plupart des sites d'habitat de l'âge du Bronze, de fonds de cabanes, de trous de poteaux, de fosses détritiques, de silos, et de fondations de maison. Beaucoup de structures sont orientées dans le sens est-ouest, nord-sud, une orientation qui a conditionné la disposition même du parcellaire au premier millénaire de notre ère, influençant le paysage local sur plusieurs siècles.

La zone fouillée révèle une occupation très dense qui se trouve à côté d'une zone artisanale dispersée où se situent deux puits. Les fondations d'une maison, des fonds de cabane ont été découverts, faisant l'objet d'une première tentative de restitution. La part de l'étude céramique est importante [Fig.2], céramologue et archéozoologue Centre de recherches archéologiques de la vallée de l'Oise (Cravo), Compiègne Cristina Gonçalves*, 


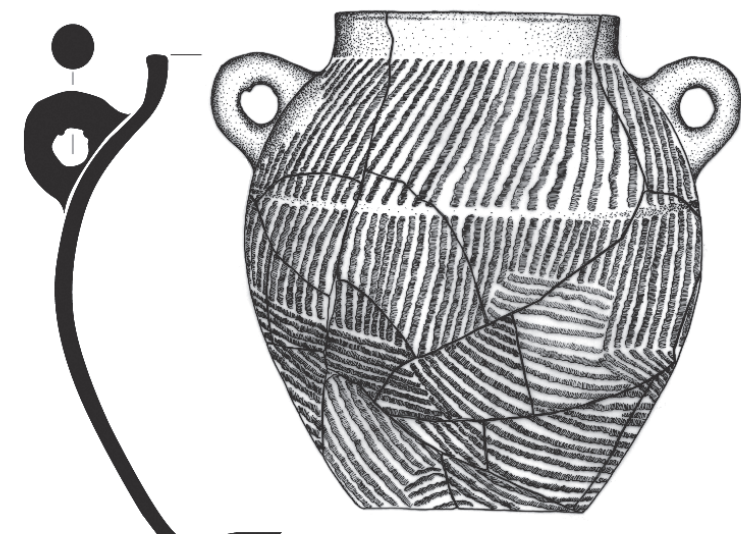

1601-34
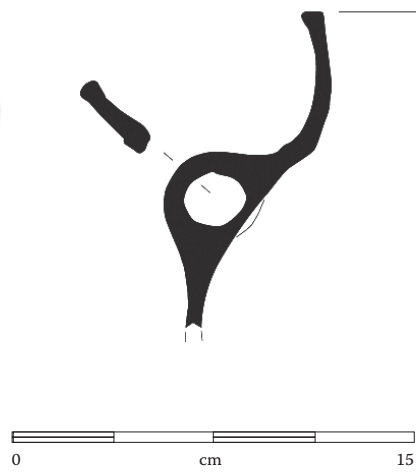

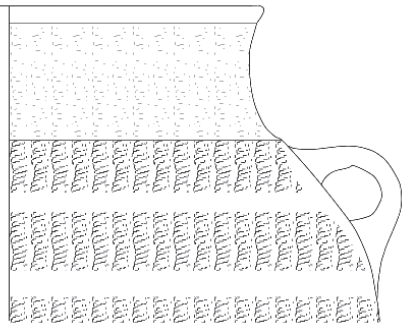

2101-11
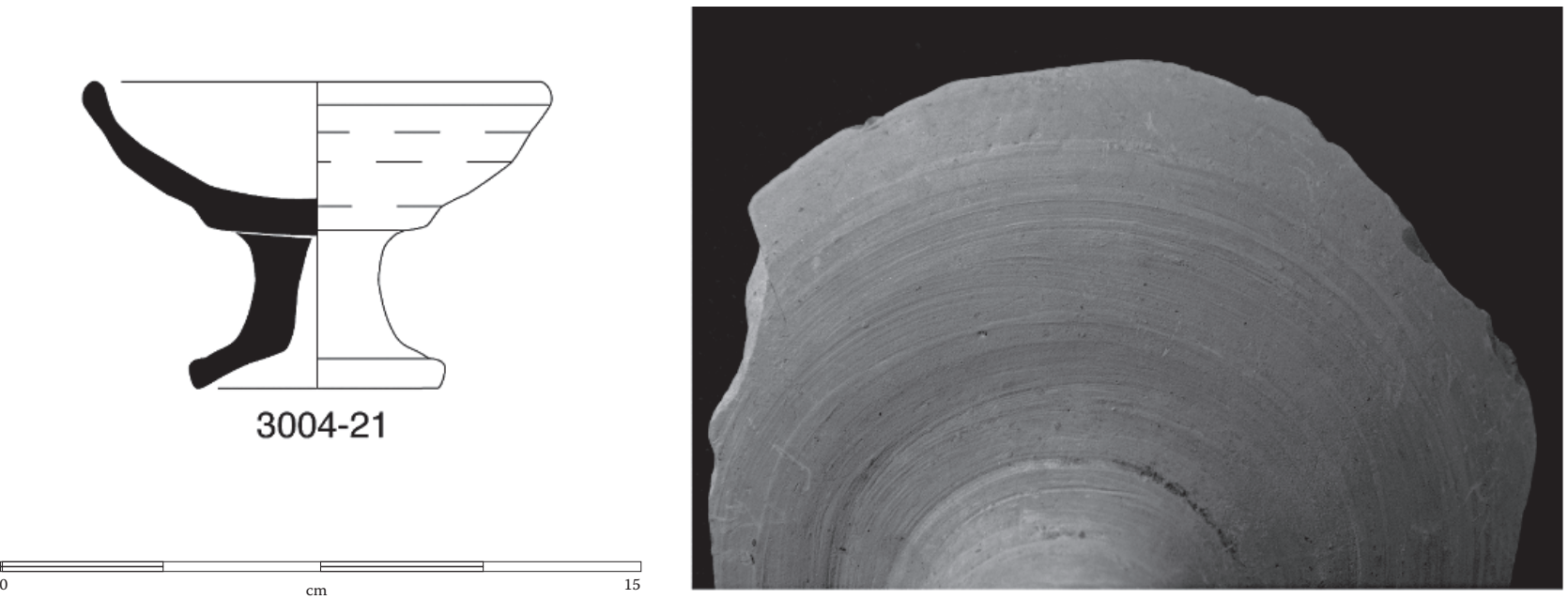

$1219-18$

[Fig.3] Cruches. Les archéologues chinois utilisent la méthode classique des dessins encrés et numérisés

(US 1601-11). Nous avons créé des trames graphiques pour rendre

visuellement les traitements de surface et les décors (US 2110-34).
[Fig.4] L'examen minutieux des coupes a permis de définir des modes

de montage par colombins

(US 3004-21) et de finitions au tour

(US 1219-18). 
et quelques aspects en sont décrits ci-dessous. Plusieurs analyses ont été entreprises, à partir des os, des graines, des pierres utilisées pour l'outillage, et d'échantillons pour la datation au carbone 14. L'étude des os animaux montre que le porc est prépondérant, puis vient le mouton, le bœuf à égalité avec le cerf, enfin le chien et le sanglier. Les espèces chassées sont d'abord le cerf, puis le sanglier et le lièvre. D'après les proportions de ces espèces, la chasse a constitué une activité très importante sur le site. L'analyse de l'ensemble des données collectées lors de la fouille ne saurait livrer tous les secrets d'un site d'habitat du début du premier millénaire à partir d'une superficie aussi petite que celle que nous avons fouillée. À travers cet exemple, nous avons cherché à montrer à nos collègues l'intérêt que présentent les méthodes spécifiques pour l'approche de ce type de site, car la méthodologie est essentielle pour apprécier la culture matérielle des villages dans son entier.

La coopération franco-chinoise menée à Gongying a révélé de part et d'autres des approches très différentes. $\mathrm{Si}$, en Chine, on étudie les villages néolithiques d'une façon beaucoup plus complète qu'on ne le fait sur les villages de l'âge du Bronze, cela tient au fait que les premiers sont en général beaucoup mieux préservés et qu'il n'y a pas la même densité d'occupation sur plusieurs siècles, plusieurs générations. De plus, la prégnance des textes pour la connaissance du passé fait qu'on néglige bien des aspects concernant la vie des villages à l'âge du Bronze.

Récemment, une découverte a soulevé un intérêt considérable. Il s'agit de fermes abandonnées lors d'une crue du fleuve Jaune au début de l'ère chrétienne. Les eaux ont recouvert une vaste région, charriant des alluvions qui se sont déposées sur plus de $7 \mathrm{~m}$ de haut. Les champs, des troncs d'arbres fossilisés, l'outillage agricole laissé sur place parce que encombrant ou lourd, les toitures affaissées sur les maisons, tout est resté quasiment dans l'état où les habitants ont laissé les lieux. Cette découverte a suscité un nouvel intérêt pour le monde agricole ancien et amènera peut-être les archéologues chinois à approfondir leurs recherches sur ce thème. Pour le moment, les études pluridisciplinaires associées aux études techniques de tous ordres ont plutôt porté sur les tombes et leur contenu. Mais les jeunes générations sont beaucoup plus ouvertes aux questions qui concernent l'homme et son environnement.

Le travail de la mission archéologique « Royaume de Chu», tout modeste qu'il est, devrait montrer l'intérêt d'associer différents spécialistes, de fouiller en utilisant des approches nouvelles. En particulier, nous avons pu mettre en évidence ce qu'apporte l'étude systématique de la céramique, sans se limiter aux seules pièces complètes, et en montrant qu'elle n'est pas seulement un marqueur chronologique, qu'il peut exister un décalage entre la céramique déposée dans les tombes, très bien étudiée en Chine, et la céramique des contextes d'habitats ruraux.
Létude du mobilier céramique. Cette mission, parallèlement à la démonstration des méthodes de fouille employées en France et principalement en archéologie préventive, nous a permis de présenter, également, les méthodes d'enregistrement et d'analyse du mobilier céramique. Notre étude s'est concentrée, principalement, sur l'enregistrement méthodique de tous les artéfacts. Elle a été effectuée par unité stratigraphique avec un décompte systématique des catégories techniques et des traitements de surface incluant les différents types de décors et les formes. Ce travail, en France, depuis des années, est réalisé sur tous les types de sites et périodes chronologiques différentes. Toutes ces informations permettent aujourd'hui d'établir des typo-chronologies de référence pour des régions données et de travailler sur les aires de diffusion des productions, qui induisent les questions géographiques, culturelles et politiques.

La phase post-fouille a donc été engagée sur place, le mobilier n'étant accessible que sur le territoire chinois, dès la première année, en 2000, et s'est achevée en Chine en 2005. Les premières années ont permis d'établir les bases de l'analyse céramique qu'il a fallu adapter au fur et à mesure de l'avancement de l'étude et de la prise de connaissance du mobilier d'une culture qui nous était inconnue.

Trois équipes de céramologues travaillant sur différentes périodes (Protohistoire, Antiquité) se sont succédé, pour des raisons de disponibilité, sur quatre années incluant des phases transitoires. Ces changements d'équipes n'auraient pu se faire sans l'application de méthodes de travail rigoureuses et communes utilisées en France depuis plusieurs années.

Nous avons travaillé sur un total de 197 unités, et cinq ensembles provenant d'interfaces de fouille, qui ont livré plus de 38 ooo tessons. Après traitement et marquage de la céramique par une équipe chinoise, des essais de remontage ont été réalisés par unité stratigraphique. Un classement a été ensuite effectué en fonction des catégories techniques qu'il a fallu définir par un examen visuel des différents types d'inclusions observés dans les pâtes et par leur importance quantitative. Ce travail a été complété en France par une série de lames minces et radiographies, analyses portant sur la composition des pâtes et les modes de façonnage à partir d'échantillons rapportés du site. Chaque type de pâte a, ensuite, été trié selon les traitements de surface et a fait l'objet d'un enregistrement individuel indiquant le nombre de tessons et le poids. Sur les fiches d'étude du mobilier apparaissent également les différents types de décors et enfin les formes. Ces dernières ont été comptabilisées, d'une part, selon la présence des bords par grande catégorie de vaisselle fournissant un nombre minimum d'individus (NMI). D'autre part, l'enregistrement des autres parties de récipients appartenant à une forme reconnue, comme, par exemple, les bases de coupes, les pieds de tripodes ou encore les fonds de pots, ont livré un nombre réel d'individus calculés par comparaison aux éléments les plus représentés. 
La base de données ainsi établie a permis de prendre en considération la totalité du mobilier exhumé sur le site côté français. À l'issue de cet enregistrement, les éléments les plus pertinents (forme, bord, décor) ont été dessinés et/ou photographiés afin d'avoir, à notre retour en France, une donnée visuelle et complète des ensembles. Les dessins « minutes » effectués au conformateur à l'échelle $1 / 1$ (outil qui a séduit les archéologues chinois), ont été repris en informatique (Adobe Illustrator) et mis à l'échelle $1 / 3$. Les photographies ainsi que les dessins précis ou estampages (effectués par une équipe chinoise) ont été intégrés aux planches [Fig.3].

Lors des deux dernières années de la mission, nous avons effectué des essais de remontage inter-unités stratigraphiques dans une même structure et entre les structures après lecture du travail portant sur l'analyse des recoupements stratigraphiques. Les nouvelles observations issues de cette phase du travail portent notamment sur le mode de montage des formes et la présence de certaines catégories techniques dans des unités stratigraphiques [Fig.4].

Létude finale du mobilier céramique a pu réellement commencer en 2007 après une longue phase de travail sur la mise au propre des planches, le regroupement des données et les enregistrements. La mise en place de la typologie par forme a été basée sur des critères formels (bord, pied, etc.) et métriques (diamètre, hauteur, etc.). Chaque partie des récipients a fait l'objet d'une description, incluant les décors, avec un classement typologique, types enregistrés sur une nouvelle base de donnée par individu ou groupes d'individus.

L'étude typo-chronologique, qui est en cours, permettra de montrer l'évolution des formes et des techniques sur plusieurs siècles et de définir le type d'occupation du site. Ce travail, dont l'un des objectifs était l'enregistrement de la totalité des informations, sera publié conjointement avec létude chinoise. Il donnera aux archéologues chinois la possibilité de travailler sur des ensembles complets référencés qui pourront servir de source à des études faites dans un cadre géographique plus large.

L'Inrap pourrait faire valoir son savoir-faire en Chine. La plus grande part du travail des archéologues chinois porte, en effet, sur quelques rares sites protégés où sont effectuées des fouilles programmées, mais essentiellement sur des fouilles de sauvetage. Récemment, avec les vastes travaux d'aménagement du barrage des Trois-Gorges, maintenant achevés, et ceux, en cours, du grand canal qui doit relier les eaux du fleuve Bleu à celles du fleuve Jaune, une démarche comparable à celle pratiquée par les archéologues de l'Inrap y devient indispensable. Car les archéologues chinois travaillent différemment, en faisant réaliser le travail de terrain par des ouvriers, pour la plupart recrutés localement et sans expérience. Proposer aux étudiants chinois d'effectuer des stages de formation au sein de l'Inrap, et même, à plus long terme, créer un chantier-école, serait grandement souhaitable. 